\title{
Does repeated dry sauna bathing change thermoregulation process in elite cross-country skiers?
}

\author{
Z. Drzazga' ${ }^{1} \cdot$ M. Binek ${ }^{1} \cdot$ I. Pokora ${ }^{2}$
}

Received: 3 July 2019 / Accepted: 4 May 2020 / Published online: 15 May 2020

(c) The Author(s) 2020

\begin{abstract}
The aim of this study was to evaluate the influence of a series of ten finnish dry sauna bathing on skin temperature following exercise test for elite cross-country skiers and test, if the regular sauna baths induce any changes in physiological or haematological parameters in elite athletes. Ten elite cross-country skiers $(21.7 \pm 3.1$ years, height: $1.79 \pm 0.025 \mathrm{~m}$, body mass: $71.06 \pm 4.77 \mathrm{~kg}$ ) participated in this study. They performed two continuous exercise tests, before and after a series of ten sauna baths. During experiment, the superficial temperature distributions, physiological and haematological parameters were measured. The marked temperature drop was visible in upper body and smaller in lower part of body after exercise test. 10 min after experimental exercise test slow return of the skin temperature to pre-workout values was observed in lower limbs unlike upper part of body. Results of this study indicated that passive induced heat acclimation have a moderate, positive effect (above 2\%) on plasma volume and a small impact on physiological and temperature responses to exercise in elite athletes. We conclude that ten finnish dry sauna baths can induce weak changes in the superficial temperature distributions following exercise test, a certain decrease in resting heart rate and small increase in plasma volume in the elite cross-country skiers.
\end{abstract}

Keywords Thermal mapping $\cdot$ Sauna stimulation $\cdot$ Thermoregulation $\cdot$ Cross-country skiers $\cdot$ Physiological assay

\section{Introduction}

Thermal imaging is a non-invasive method used to visualization human body temperature changes in response to physiological processes and may be useful to detect physiological and pathophysiological reactions related to the control of the skin temperature. Recently, this method is most often used to monitor individual muscles to detect overloads and injuries as well as to monitor the body temperature distributions during and after exercise [1-6]. A few reports also suggested that MIT (medical infrared thermography) may be useful for detection effectiveness of skin heating or cooling and as a convenient and effective diagnostic tool for detection of

\section{Z. Drzazga}

zofia.k.drzazga@gmail.com

1 University of Silesia in Katowice, Faculty of Science and Technology, The Silesian Centre for Education and Interdisciplinary Research, ul. 75 Pułku Piechoty 1A, 41-500 Chorzów, Poland

2 Department of Physiological and Medical Sciences, Institute of Sport Sciences, The Jerzy Kukuczka Academy of Physical Education in Katowice, Katowice, Poland peripheral vascular disorders [7]. It has been hypothesized that the MIT can provide excellent additional information about the existence of some differences in thermal behaviour of the skin after exercise, in athletes who were using a series of sauna baths.

In the field of physical activity and sport, it is important to optimize the recovery process of the athletes. Sauna bathing is a form of passive heat therapy which can be routinely used as a non-pharmacological treatment regimen in certain medical disorders such as cardiovascular diseases [8-10] or anorexia [11] to relieve symptoms and improve wellness.

Finnish sauna has a substantial effect on the whole human body. Alternate hot and cold conditions used in sauna bathing are considered to accelerate biomedical athletic recovery and are frequently used as therapies in sport, recreation and rehabilitation [12]. This treatment is supposed to help in regeneration as well as increase the overall efficiency of the athletes. Several studies have documented the positive effect of sauna on health of participant [13-16]. Influence of sauna on human body is mainly associated with an increase in the thermoregulatory and circulatory demands. In order to maintain thermal homoeostasis, the body actively responds to the thermoregulatory mechanisms. For sports requiring high endurance, the 
sauna is a complementary biological regeneration treatment. Regular exposure to passive heating (e.g. sauna) induces cardiovascular and sweating changes: positive heat adaptation. Scoon et al. [15] as well as Ernst et al. [17] showed that a 3 -week post-exercise sauna bathing produced a worthwhile enhancement of endurance running performance, probably by increasing blood volume. Complete heat acclimation (HA) requires up to 14 days, but the systems of the body adapt to heat exposure at varying rates. The early adaptations (initial 1-5 days) involve an improved control of cardiovascular function, including expanded plasma volume, reduced heart rate and autonomic nervous system habituation which redirects cardiac output to skin capillary beds and active muscle. These adaptations contribute to improvements in cardiovascular stability, sweating capacity and thermoregulation processes that work in combination to improve physical capacities and sports performance in the heat $[18,19]$ and may also improve aerobic performance in cooler conditions up to 6\% [20]. To date, heat acclimation has been achieved via both active strategies [20] and passive strategies [21] and as short ( $<7$ days)-, medium (8-14 days)-, and long ( $>14$ days)-term heat adaptation protocols [18]. Short-term heat acclimation may be the preferred regime for highly trained athletes, as it potentially provides less disruption of quality training [22], while medium-term protocols elicit greater physiological adaptations and performance improvements [23].

Cross-country skiing is one of the most demanding sports aimed at endurance which requires one of the highest aerobic fitness levels. For sports requiring high endurance, the sauna is a complementary biological regeneration treatment. The change associated with heat acclimation such as the hypervolemic response of the blood plasma due to a transient reduction in haemoglobin and haematocrit ratios is expected after HA. We also supposed that HA could affect normal thermal patterns in the study in which the athletes were exposed to constant exercise. For that reason, the aim of this study was to evaluate the influence of a series of ten finnish dry sauna bathing on skin temperatures following exercise test for elite cross-country skiers. It is interesting to check if MHA induce bigger change in temperature distribution in muscles of upper or lower part of body. What is more, we try to study, if the regular sauna baths may lead to significantly reduced heart rate before or after experimental exercise as well as expanded plasma volume (PV), which could indicate the marked development of heat adaptation (MHA) in elite athletes.

\section{Materials and methods}

\section{Subjects}

The study group was elite male cross-country skiers $(n=10)$, recruited from sports associations (AZS) the Jerzy Kukuczka Academy of Physical Education) who had similar training experience ( $>10$ years); age: $21.7 \pm 3.1$ years, height: $1.79 \pm 0.025 \mathrm{~m}$, body mass: $71.06 \pm 4.77 \mathrm{~kg}$, max. oxygen uptake: $\mathrm{VO}_{2 \max }=4.49 \pm 0.5 \mathrm{~L} \mathrm{~min}^{-1}$, BMI: $22.43 \pm 1.54 \mathrm{~kg}$ $\mathrm{m}^{-2}$, body fat: $8.11 \pm 3.22 \%$. Participants were instructed to avoid alcohol, smoking, caffeine, large meals, cosmetics before the assessment. Volunteers were in the transitional period (April/May)of the annual training plan. They were informed of the purpose, possible risks and the benefits of the study before giving their written consent to participate. The study protocol conformed to the ethical guidelines of the World Medical Association Declaration of Helsinki and was approved by the Ethics Committee of the Jerzy Kukuczka Academy of Physical Education in Katowice. Subjects were excluded if they had a concurrent cardiovascular or respiratory disorder. Data from all completed measurements were analysed statistically.

\section{Preliminary study}

The study protocol was divided into three visits to the laboratory. During the first visit, 1 week prior to the start of the experiment, all subjects performed a standard incremental exercise test to measure their individual aerobic capacity $\left(\mathrm{VO}_{2} \max \right)$ and individual anaerobic threshold (PPA). PPA was determined for each participant. Details of preliminary testing for determining of the mention values were described in [5]. The PPA had been used to calculate target work rates for submaximal exercise testing, which were using during experimental tests.

\section{Experiment tests}

The subjects performed the same continuous exercise test twice, before and after a series of ten sauna baths. Experimental continuous exercise test (EET test) consisted of $\sim 60$ min running with the intensity below the individual PPA lactate threshold (calculated individually for each athlete during the preliminary study) on treadmill H/P/Cosmos Pulsar at $20 \pm 1{ }^{\circ} \mathrm{C}$ ambient temperature, $56 \pm 3 \%$ relative humidity and $985 \pm 5 \mathrm{hPa}$ atmospheric pressure, in a human functional testing laboratory, Academy of Physical Education in Katowice. To avoid the effects of diurnal variations of body temperature, exercise tests were always performed in the morning at the similar time. It should be noted that EET test consisted of $\sim 60 \mathrm{~min}$ running seems to be correlated 
with the working body parts in cross-country skiers what was reflected on the thermal map.

\section{Sauna baths}

Subjects completed heat acclimation sessions consisting of ten Finnish sauna baths with a 2 days rest between 5 and 6 heat exposures (medium-term heat adaptation, MHA). Participants spent a total of $45 \mathrm{~min}$ in sauna (sauna bathing per day, an average temperature at face height $90^{\circ} \pm 2{ }^{\circ} \mathrm{C}$, and average relative humidity $12 \pm 4 \%$ ). This time was divided into three parts (each $15 \mathrm{~min}$ ) and separated by two 4-6 $\mathrm{min}$ showers to cool the body. All sauna baths were always conducted in the afternoon at the same period.

First experimental exercise was performed before heat exposure (control). The second one was performed $12 \mathrm{~h}$ after completed the series of ten heat exposures (MHA).

\section{Thermal imaging}

Thermal images were recorded in accordance with the Glamorgan Protocol [24] taking into consideration checklist directed at standardizing thermographic imaging in sports and exercise medicine [25]. Thermal maps of face, upper part of body at front and back, thighs, knees and calves at front and from the rear were taken for each participant before, immediately after exercise and at $10 \mathrm{~min}$ of recovery using camera Flir Systems E60 with resolution $320 \times 240$ pixels, sensitivity $0.05 \mathrm{~K}$. Camera was calibrated by black body, and the emissivity was set at the range of 0.97-0.98. During imaging, camera was positioned perpendicular to the region of interest at about $1 \mathrm{~m}$ distance between object and camera. The data ROI were analysed by using a ThermaCAM TM Researcher Pro 2.8 SR-3 focusing on the muscle zones following to the model proposed by FernandezCuevas et al. [2].

\section{Physiological parameters}

Before and during EET tests, heart rate (HR) (PE-3000 Sport-tester, Polar Inc., Finland) and oxygen uptake $\left(\mathrm{VO}_{2}\right)$ (Oxycon RALPHA (Jaeger, Germany) were recorded every $10 \mathrm{~min}$.

\section{Blood collections}

Samples of venous blood for haemoglobin $[\mathrm{Hb}]$ and haematocrit [Hct] assessment were taken at rest before exercise tests (Rest) and immediately afterwards. To assay haemoglobin $(\mathrm{Hb})$, standard cyan-methaemoglobin method was used. (HG980, Randox, UK). Changes in the concentration of haemoglobin $(\mathrm{Hb})$ and haematocrit $(\mathrm{Hct})$ were used to determine the relative change in plasma volume described by Dill and Costill [26].

Statistical analysis was performed in Statistica 12 software. All data were checked for normality using Shapiro-Wilk test, and then, data were analysed using $t$ tests, if it was necessary nonparametric Mann-Whitney test. Temperature parameters were analysed using one-way ANOVA repeated measurements. The homogeneity of variances was verified by Leven' test. Statistical significance between the means was set at $p<0.05$. The effect size was also estimated in assessment of surface temperature distribution in body using ANOVA test.

\section{Results}

Example of thermographic recordings of body surface including upper and lower part, before, immediately after and 10 min after experimental exercise for ski-runners after 10 sauna bathing cycle (after MHA) is shown in Fig. 1.

One can see that skin temperature changes in response to exercise of upper and lower part of body shows different character. The marked temperature drop after experimental exercise was visible in upper body; in lower part of body, temperature changes were smaller and generally opposite. An explicit increase in temperature in thighs, knees was observed and weaker for calves. After 10 min recovery, the skin temperature of upper part of body decreased far, while the temperature of legs muscles slowly returned to pre-workout values.

Mean values of temperatures: T1-before exercise, T2 -immediately after exercise, T3-10 min of recovery for selected muscle zones, before and after adaptation to heat are listed at Table 1. More statistical significant drops in skin temperature after EET test were found for rear upper part of body than upper part at front, especially after $10 \mathrm{~min}$ of recovery. It is interesting that after MHA we observed similar skin temperature decrease immediately after exercise. Significant increase in temperature in vastus lateralis and knee (at front) was obtained immediately after EET test, but it disappeared $10 \mathrm{~min}$ later. Moreover, it should be noted that partial eta-squared $\eta_{\mathrm{p}}^{2}$ was in range in "medium" effect size for significant changes of temperature (Table 1).

In order to get better insight into thermoregulation process, changes in skin body temperatures with respect to effort and restitution before and after adaptation to heat were analysed. Differences of mean skin temperature between temperature immediately after and before exercise $\Delta T_{2-1}=T_{2}-T_{1}$ as well as between temperature $10 \mathrm{~min}$ after and before exercise $\Delta T_{3-1}=\left(T_{3}-T_{1}\right)$ over selected muscle zones (21 ROIs) of sportsmen before and after sauna bathing cycle were calculated and presented in Fig. 2a, b. Change in skin temperature response to the effort before and after 
(a) Before training
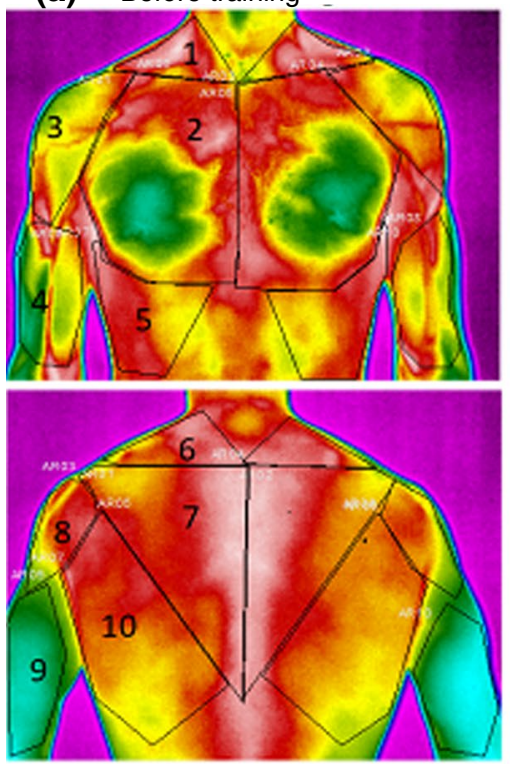

(b)
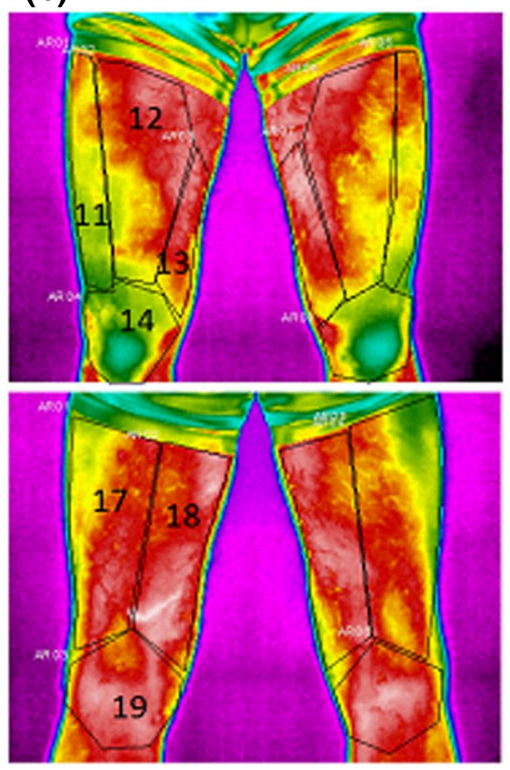

Immidietly after training
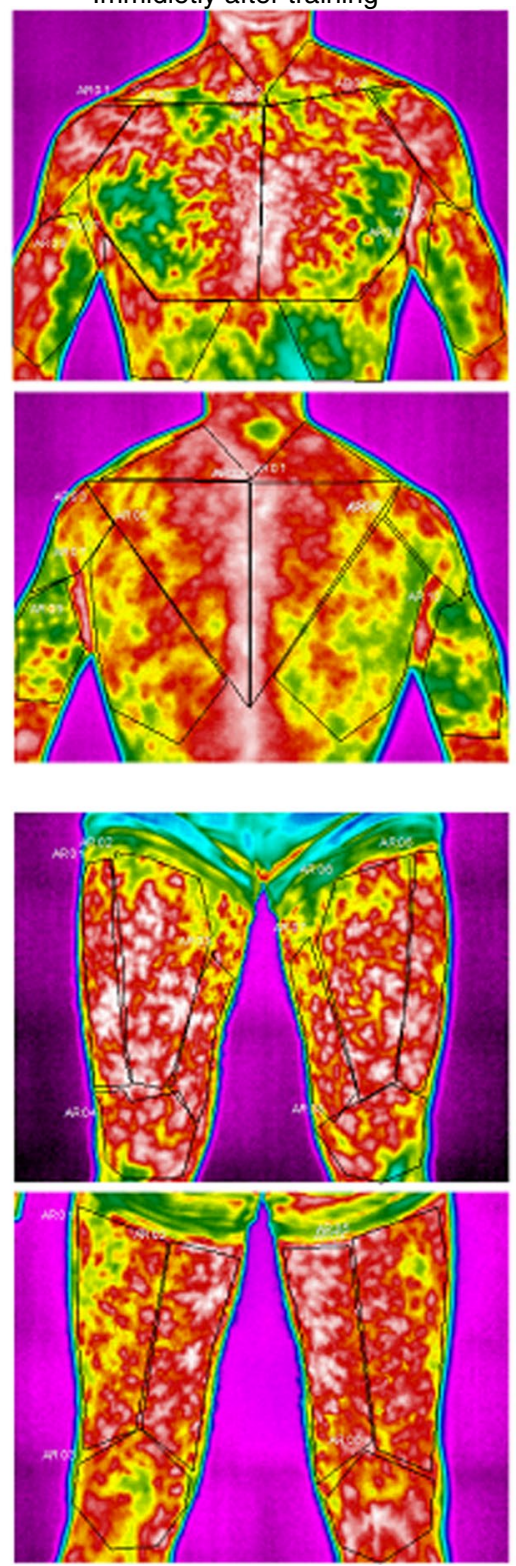

10 min after training
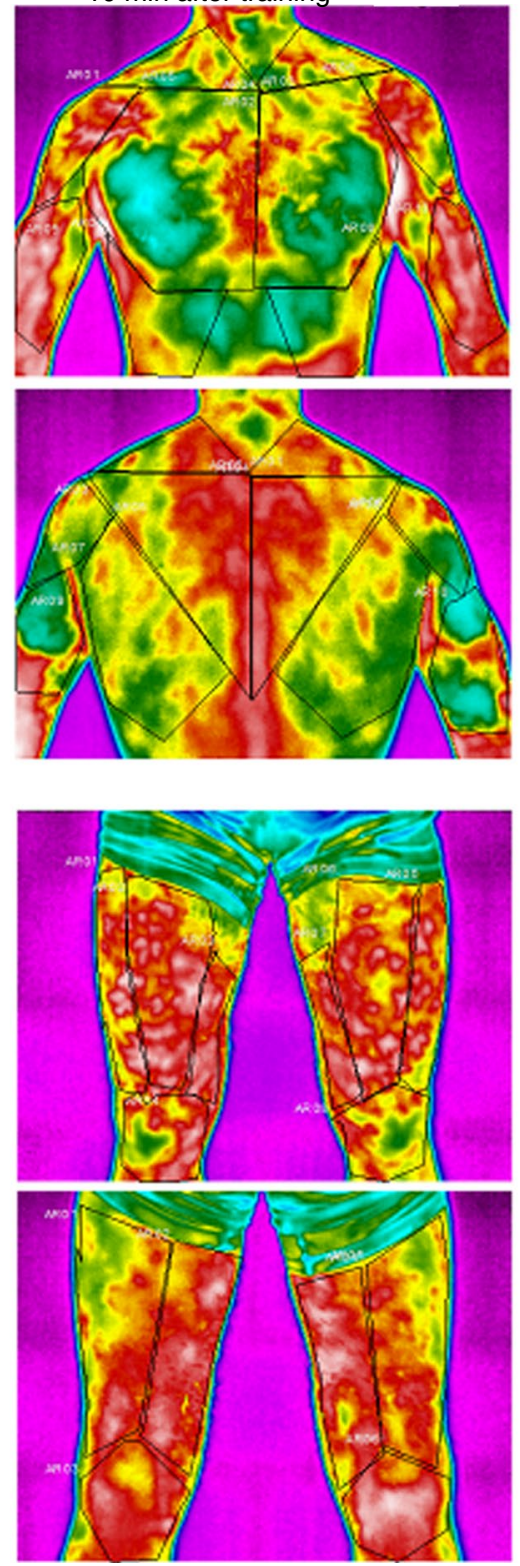
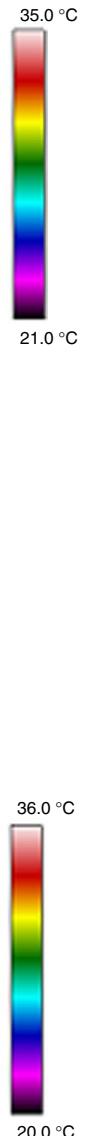

Fig. 1 Thermal mapping of upper body and things muscles during experimental exercise for sportsman after 10 sauna bathing cycle. Muscles of upper and lower part of body (front and rear) are presented (1-trapezius muscle, 2-pectoralis major, 3-deltoid muscle, 4-biceps brachii, 5-serratus anterior, 6-trapezius muscle (neck),

adaptation to heat is week. Figure 2 reveals certain, not significant differences in skin temperature $\Delta T_{2-1}=T_{2}-T_{1}$ over several muscles (Vastus lateralis, Rectus femoris, Knee (at front), Soleus). However, they disappeared with restitution period. Plots of $\Delta T_{3-1}$ before and after MHA are nearly the same for the studied muscle zones beside trapezius muscle (back) and rear of deltoid muscle. Relatively, the biggest 7-trapezius muscle (back), 8-rear of deltoid muscle, 9-triceps brachii, 10-latissimus dorsi, 11-vastus lateralis, 12-rectus femoris, 13-vastus medialis, 14-knee (at front), 17-biceps femoris, 18 - rest of thigh muscle, 19-knee (rear)

change in temperature observed immediately after exercise in vastus lateralis and knee at front seems to be due to intensive activity of legs in cross-skiers. On the other hand, the deepening of temperature changes over trapezius muscle (back) and rear of deltoid muscle indicates increased cooling of the torso(back) by sweat evaporation caused by effort.

Physiological and haematological markers assessed at rest $(0)$ and at the end $(T)$ of exercise test, before and after series of 10 sauna baths treatment are presented in Table 2 . 
Table 1 Temperature values obtained in experiments T1—before exercise, T2-immediately after exercise, T3—after 10 min of restitution for selected muscle zones before and after sauna cycle

\begin{tabular}{lllll}
\hline Muscle zone & $\begin{array}{l}\text { Stage of } \\
\text { experiment }\end{array}$ & Mean $T_{1} \pm \mathrm{SD} /{ }^{\circ} \mathrm{C}$ & Mean $T_{2} \pm \mathrm{SD} /{ }^{\circ} \mathrm{C}$ & $\mathrm{Mean} T_{3} \pm \mathrm{SD} /{ }^{\circ} \mathrm{C}$ \\
\hline Trapezius muscle & Before & $34.41 \pm 0.58$ & $\mathbf{3 3 . 0 0} \pm \mathbf{1 . 2 0} ; p=0.04 ;\left(\eta_{\mathrm{p}}^{2}=0.36\right)$ & $\mathbf{3 3 . 1 0} \pm \mathbf{0 . 7 4} ; p=0.01 ;\left(\eta_{\mathrm{p}}^{2}=0.49\right)$ \\
& After & $34.33 \pm 0.59$ & $33.17 \pm 1.19$ & $\mathbf{3 3 . 0 8} \pm \mathbf{1 . 3 4} ; p=0.05 ;\left(\eta_{\mathrm{p}}^{2}=0.34\right)$ \\
Pectoralis major & Before & $33.93 \pm 0.72$ & $33.07 \pm 1.54$ & $\mathbf{3 2 . 6 0} \pm \mathbf{1 . 0 5} ; p=0.04 ;\left(\eta_{\mathrm{p}}^{2}=0.34\right)$ \\
& After & $34.03 \pm 0.95$ & $33.16 \pm 1.04$ & $32.63 \pm 1.50$ \\
Serratus anterior & Before & $33.79 \pm 0.77$ & $32.80 \pm 1.41$ & $\mathbf{3 2 . 5 0} \pm \mathbf{0 . 9 9} ; p=0.04 ;\left(\eta_{\mathrm{p}}^{2}=0.36\right)$ \\
& After & $34.26 \pm 0.93$ & $33.05 \pm 1.03$ & $\mathbf{3 2 . 6 4} \pm \mathbf{1 . 4 6} ; p=0.05 ;\left(\eta_{\mathrm{p}}^{2}=0.38\right)$ \\
Trapezius muscle (neck) & Before & $34.52 \pm 0.48$ & $\mathbf{3 3 . 7 1} \pm \mathbf{0 . 5 9} ; p=0.04 ;\left(\eta_{\mathrm{p}}^{2}=0.36\right)$ & $\mathbf{3 3 . 2} \pm \mathbf{0 . 8 3} ; p=0.01 ;\left(\eta_{\mathrm{p}}^{2}=0.50\right)$ \\
& After & $34.56 \pm 0.27$ & $\mathbf{3 3 . 7 3} \pm \mathbf{0 . 5 8} ; p=0.02 ;\left(\eta_{\mathrm{p}}^{2}=0.50\right)$ & $\mathbf{3 2 . 8 8} \pm \mathbf{1 . 3 0} ; p=0.01 ;\left(\eta_{\mathrm{p}}^{2}=0.53\right)$ \\
Trapezius muscle (back) & Before & $33.92 \pm 0.32$ & $33.53 \pm 0.64$ & $\mathbf{3 2 . 8 0} \pm \mathbf{0 . 8 0} ; p=0.01 ;\left(\eta_{\mathrm{p}}^{2}=0.50\right)$ \\
& After & $34.55 \pm 0.21$ & $\mathbf{3 3 . 7 5} \pm \mathbf{0 . 6 9} ; p=0.04 ;\left(\eta_{\mathrm{p}}^{2}=0.42\right)$ & $\mathbf{3 2 . 8 6} \pm \mathbf{1 . 4 9} ; p=0.03 ;\left(\eta_{\mathrm{p}}^{2}=0.47\right)$ \\
Latissimus dorsi & Before & $33.56 \pm 0.44$ & $32.80 \pm 0.92$ & $\mathbf{3 2 . 3 0} \pm \mathbf{0 . 6 8} ; p=0.01 ;\left(\eta_{\mathrm{p}}^{2}=0.53\right)$ \\
& After & $33.91 \pm 0.25$ & $\mathbf{3 3 . 1 2} \pm \mathbf{0 . 5 9} ; p=0.03 ;\left(\eta_{\mathrm{p}}^{2}=0.47\right)$ & $\mathbf{3 2 . 5 1} \pm \mathbf{1 . 3 8} ; p=0.04 ;\left(\eta_{\mathrm{p}}^{2}=0.42\right)$ \\
Biceps & Before & $33.54 \pm 0.66$ & $\mathbf{3 2 . 6 1} \pm \mathbf{0 . 7 0} ; p=0.05 ;\left(\eta_{\mathrm{p}}^{2}=0.32\right)$ & $32.90 \pm 0.77$ \\
& After & $33.40 \pm 0.38$ & $32.73 \pm 0.71$ & $32.92 \pm 0.58$ \\
Vastus Lateralis & Before & $31.48 \pm 0.49$ & $32.23 \pm 0.70$ & $31.50 \pm 0.99$ \\
& After & $31.48 \pm 1.01$ & $\mathbf{3 3 . 1 6} \pm \mathbf{0 . 7 6} ; p=0.02 ;\left(\eta_{\mathrm{p}}^{2}=0.51\right)$ & $31.76 \pm 1.17$ \\
Knee(at front) & Before & $29.85 \pm 0.81$ & $\mathbf{3 1 . 9 7} \pm \mathbf{1 . 1 2} ; p=0.01 ;\left(\eta_{\mathrm{p}}^{2}=0.54\right)$ & $30.80 \pm 1.04$ \\
& After & $29.78 \pm 0.65$ & $\mathbf{3 2 . 5 9} \pm \mathbf{1 . 4 2} ; p=0.00 ;\left(\eta_{\mathrm{p}}^{2}=0.66\right)$ & $30.91 \pm 1.52$
\end{tabular}

Only results for muscles which statistical significant differences between $T_{2}$ and $T_{1}$ or $T_{3}-T_{1}$ are listed ( $p \leq 0.05$ (bold) and partial eta-squared $\left.\eta_{\mathrm{p}}^{2}>0.3\right)$

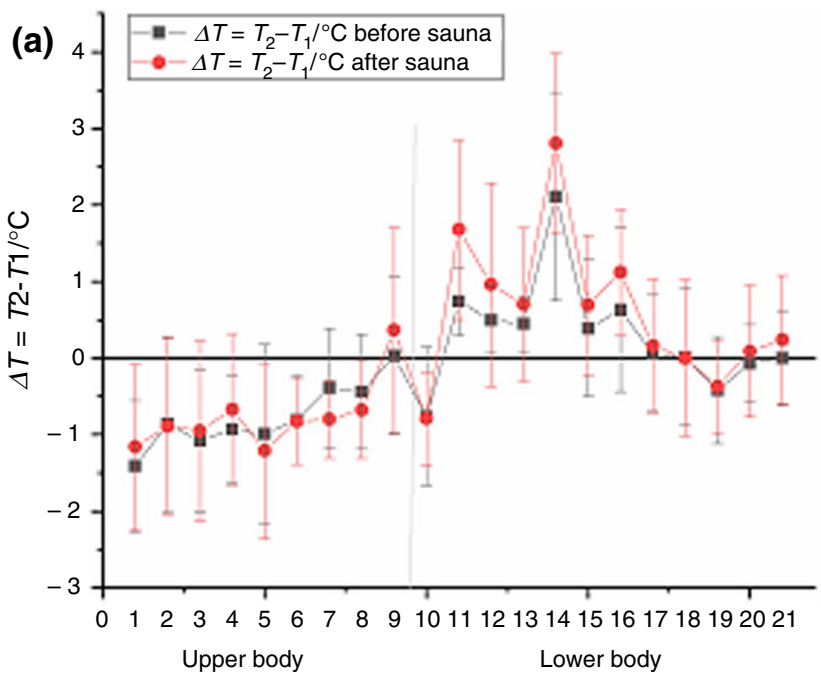

Muscle zone

Fig. 2 Changes in skin body temperatures with respect to effort and restitution before and after adaptation to heat. 1-trapezius muscle, 2-pectoralis major, 3-deltoid muscle, 4-biceps brachii, 5-serratus anterior, 6-trapezius muscle(neck), 7-trapezius muscle (back), 8-rear of deltoid muscle, 9-triceps brachii, 10-latissimus dorsi,

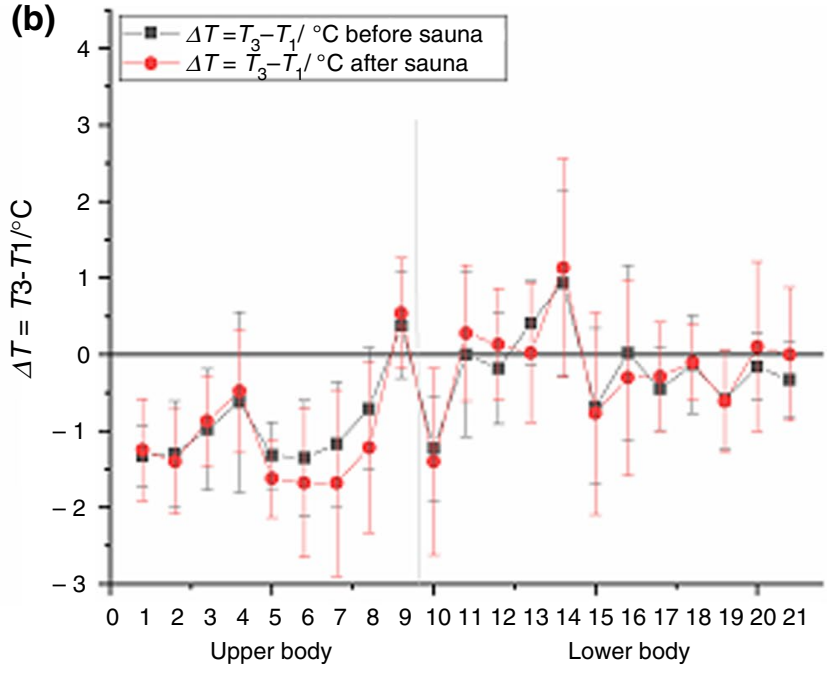

Muscle zone

11-vastus lateralis, 12-rectus femoris, 13-vastus medialis, 14knee (at front), 15-tibialis anterior, 16-soleus, 17-biceps femoris, 18-rest of thigh muscle, 19-knee (rear), 20-gastrocnemius caput laterale, 21-gastrocnemius caput mediale 
Table 2 Physiological and haematological markers assessed at rest $(0)$ and at the end $(T)$ of exercise test, before (control) and after series of 10 sauna baths treatment (MHA)

\begin{tabular}{lcr}
\hline Variable & \multicolumn{1}{c}{ Control } & \multicolumn{1}{c}{ After MHA } \\
\hline $\mathrm{HR}_{\text {rest }} /$ beats $\mathrm{min}^{-1}$ & $82.46 \pm 12.93$ & $76.42 \pm 12.69$ \\
$\mathrm{HR}_{\text {max }} /$ beats $\mathrm{min}^{-1}$ & $180.29 \pm 11.63$ & $177.7 \pm 5.24$ \\
$\mathrm{WR}_{\mathrm{T}} / \mathrm{W}$ & $262 \pm 25.19$ & $260 \pm 25.44$ \\
$\mathrm{VO}_{2} / \mathrm{L} \mathrm{min}$ & -1 & $3.50 \pm 0.62$ \\
$\Delta \mathrm{PV} / \%$ & $3.58 \pm 0.47$ & $+2.13 \pm 1.15$ \\
$\mathrm{Hb}_{0} / \mathrm{g} \mathrm{dL}^{-1}$ & & $14.75 \pm 0.78$ \\
$\mathrm{Hb}_{\mathrm{T}} / \mathrm{g} \mathrm{dL}^{-1}$ & $15.15 \pm 1.01$ & $15.2 \pm 0.69$ \\
\hline
\end{tabular}

$\mathrm{HR}_{\text {rest }}$ - heart rate in rest state; $\mathrm{HR}_{\max }$ - terminal heart rate; $\mathrm{WR}_{\mathrm{T}}$ terminal work rate; $\mathrm{VO}_{2}$ - oxygen uptake; $\Delta \mathrm{PV} \%$ - change in plasma volume; $\mathrm{Hb}_{0}, \mathrm{Hb}_{\mathrm{T}}$ - basal and terminal of the blood haemoglobin concentration, respectively

Any statistical significant change was obtained in studied parameters. However, one should note a some decrease in $\mathrm{HR}_{\text {rest }}$ and hemoglobin concentration after 10 sauna baths compared to control conditions. Moreover, calculation indicated that regular sauna baths can have a moderate, positive effect on resting plasma volume $(\Delta \mathrm{PV}+2.13 \%)$.

\section{Discussion}

Results of this study indicated that passive heat acclimation (MHA) induced a certain impact on selected physiological/ haematological parameters and skin temperature.

Prediction of EET test was that it strongly affected on skin temperature over muscles of lower and upper limbs. However, interesting and bigger changes occurred in torso, especially on back.

The drop of skin temperature over the selected regions of the upper part of body seems to be associated with cooling effect of sweat evaporation. Sweating is the most important thermoregulatory reaction by which heat balance is maintained following a rise in body temperature during endogenous exercise-induced heat load. During exercise, the highest evaporative heat loss following take place from back and chest [27], what was also observed in our study of cross-skiers.

It is known that heat dissipation, under conditions of exercise-thermal stress, is mediated primarily by evaporation of sweat. Heat acclimation has been shown to enhance sweat production by eliciting changes in the sensitivity of eccrine glands, total sweat output and distribution of gland activity. The physical training induces peripheral adaptations, while heat acclimation produces both peripheral and central modifications. It is suggested that repeated cutaneous heat stimulation may be essential to the development of central sudomotor changes [28, 29].
Untrained non-heat acclimated individuals are able to physiologically wet a maximum of approximately $72 \%$ of their skin surface. Increasing the number of sweat glands activated per surface area permit a more complete saturation of the skin surface and a great evaporation. Physiologically, the ability to achieve the better dynamics of sweating an improved acclimation status facilitated by a higher local sweating rate on both the arm and back mediated by a greater number of simultaneously activated sweat glands, which potentially facilitated an enhanced distribution of sweat on the skin surface and the attainment of greater rates of maximal evaporative heat loss [30].

Tyler et al. [31] have shown that resting temperature of skin is unaffected by HA of any duration, but HA has a large effect on reducing mean $T$ skin during exercise. In our study, despite the lack of MHA effect on resting temperature $\left(T_{1}\right)$ there was a large effect of MHA on reducing mean superficial temperature over muscle zones after exercise in upper part of body (Table 1). According to our previous article, the marked skin temperature drop after similar exercise was visible in upper body in cross-country skiers and in swimmers in lower part of body [5], indicating that as changed to surface temperature distribution depends on the type of sport practiced.

It was reported that normal skin temperature pattern is affected for many hours after exercise more in some areas than others, depending on the activity of the structures below the ROI and, in a different way, according to the type of performed exercise [2]. Our studies show that repeated sauna baths a slight influence on the pattern of skin temperature changes with respect to effort and restitution in elite athletes. Character of plots of changes in skin body temperatures is the same, but its amplitude is slightly different $\Delta T_{2-1}$ and $\Delta T_{3-1}$ in the anterior part of lower extremities (knees, vastus lateralis) and for torso back (trapezius muscle neck and back), respectively (Fig. 2).

We also showed that regular sauna baths had a moderate, positive effect on increasing resting PV $(+2.13 \%)$. Data from meta-analysis [32] indicated that the size of PV increase after HA was influenced by the number of days of heat exposure the hydration state of the person. In trained men, daily post-exercise sauna bathing for 10-15 thirty minute sessions over $2-3$ weeks $\left(80-100{ }^{\circ} \mathrm{C}, 10-20 \% \mathrm{RH}\right)$ caused an increase plasma volume $7-18 \%$ [15].

The MHA did not alter $\mathrm{VO}_{2}$; however, some reduction in rest HR after MHA was observed by us (Table 2). Willmott et al. [33] indicated no statistically significant changes HR in resting or after short-term HA. However, a significant heart rate decreases of $4 \%$ (after twice daily heat stress exposure for 2 days) and 6\% (after once daily heat exposure for 4 days) was demonstrated during exercise. These authors reported that HR adjustment was typically concurrent with hypervolemia. 
Application of sauna bathing changes only a little thermoregulatory process in elite athletes. Probably, the endurance athletes are exposed to endogenous thermal load, potentially constraining adaptation to heat during training process [18-21]. There are relative small information about MHA effect on vasodilatation, vasoconstriction and sweat evaporation to exercise in professional athletes. Therefore, further studies are needed in order to understand the mechanisms of thermoregulation, especially taking into account thermal stimulations using in biological regeneration of sportsmen as well as in medical treatment.

The main limitation of our study was the small number of participants recruited from the elite athletes. The second limitation could be relatively large variability in body sensitivity on sauna baths among the subjects.

\section{Conclusions}

We indicated that ten finnish dry sauna bathing had a weak impact on skin temperatures following exercise test in elite cross-country skiers. The marked drop of temperature was observed in upper part of body and was maintained during recovery. Apart from regular sauna baths induced small physiological and haematological changes, which were revealed in certain decrease in heart rate and a little increase in resting plasma volume in elite athletes.

Our research is to show that sauna treatment has little effect on the thermoregulation process in the cross-country skiers.

Acknowledgements This study was supported by statutory funds of University of Silesia in Katowice, Poland and also partly by grant Ministry of Science and Higher Education (Poland)/Nr0050/RS4/2016/54.

\section{Compliance with ethical standards}

Conflict of interest The authors declare no conflicts of interest.

Open Access This article is licensed under a Creative Commons Attribution 4.0 International License, which permits use, sharing, adaptation, distribution and reproduction in any medium or format, as long as you give appropriate credit to the original author(s) and the source, provide a link to the Creative Commons licence, and indicate if changes were made. The images or other third party material in this article are included in the article's Creative Commons licence, unless indicated otherwise in a credit line to the material. If material is not included in the article's Creative Commons licence and your intended use is not permitted by statutory regulation or exceeds the permitted use, you will need to obtain permission directly from the copyright holder. To view a copy of this licence, visit http://creativecommons.org/licenses/by/4.0/.

\section{References}

1. Marins JCB, Fernandez-Cuevas I, Arnaiz-Lastras J, Fernandes AA, Sillero-Quintana M. Applications of infrared thermography in sports. A review. Rev Int Med Cien Activ Fisica Deporte. 2015;15(60):805-24.

2. Fernandez-Cuevas I, Sillero-Quintana M, Garcia-Conception MA, Ribot-Serrano J, Gomez-Carmona P. Monitoring skin thermal response to training with infrared thermography. New Stud Athl. 2014;29(1):57-71.

3. Hildebrandt C, Zeilberger K, Francis E, Ring J, Raschner C. The application of medical infrared thermography in sports medicine. In: Zaslav KR, editor. An international perspective on topics in sports medicine and sports injury. Rijeka: InTech; 2012. ISBN 978-953-51-0005-8.

4. Arfaoui A, Polidori G, Taiar R, Popa C. Infrared thermography in sports activity. In: Prakash RV, editor. Infrared thermography. Rijeka: InTech; 2012. ISBN 978-953-51-0242-7.

5. Drzazga Z, Binek M, Pokora I, Sadowska-Krępa E. A preliminary study on infrared thermal imaging of cross-country skiers and swimmers subjected to endurance exercise. J Therm Anal Calorim. 2018;134:701-10. https://doi.org/10.1007/s1097 3-018-7311-y.

6. Cholewka A, Kasprzyk T, Stanek A, Sieroń-Stołtny K, Drzazga Z. May thermal imaging be useful in cyclist endurance tests? J Therm Anal Calorim. 2016;12:1155-62. https://doi.org/10.1007/ s10973-016-5453-3.

7. Bagavathiappan S, Saravanan T, Philip J, Jayakumar T, Raj B, Karunanithi R, Panicker TM, Korath MP, Jagadeesan K. Infrared thermal imaging for detection of peripheral vascular disorders. J Med Phys. 2009;34(1):43-7. https://doi.org/10.4103/0971-6203.

8. Kihara T, et al. Effects of repeated sauna treatment on ventricular arrhythmias in patients with chronic heart failure. Circ J. 2004;68:1146-51.

9. Kihara $\mathrm{T}$, et al. Repeated sauna treatment improves vascular endothelial and cardiac function in patients with chronic heart failure. J Am Coll Cardiol. 2002;39(5):754-9.

10. Imamura $\mathbf{M}$, et al. Repeated thermal therapy improves impaired vascular endothelial function in patients with coronary risk factors. JACC. 2001;38(4):1083-8.

11. Vähäsoini A, Vazquez R, Birmingham CL, Gutierrez E. Unreported sauna use in anorexia nervosa: evidence from the worldwide-web. Eat Weight Disord. 2004;9:50. https://doi.org/10.1007/ BF03325045.

12. Pilch W, Pokora I, Szyguła Z, Pałka T, Pilch P, Cisoń T, Malik L, Wiecha S. Effect of a single finnish sauna session on white blood cell profile and cortisol levels in athletes and non-athletes. J Hum Kinet. 2013;39:127-35. https://doi.org/10.2478/hukin-2013-0075.

13. Niewiadomy P, Tiffert-Tłok J, Szuścik K, Stolecka-Warzecha A, Kwaśna K. Estimation of changes of lower limbs reactivity among mountain cyclist under the influence of sauna treatment. Physiother Health Activ. 2016;24:12-8. https://doi.org/10.1515/ pha-2016-0003.

14. Cuddy JS, Hailes WS, Ruby BC. A reduced core to skin temperature gradient, not a critical core temperature, affects aerobic capacity in the heat. J Therm Biol. 2014;43:7-12.

15. Scoon GSM, Hopkins WG, Mayhew S, Cotter JD. Effect of postexercise sauna bathing on the endurance performance of competitive male runners. J Sci Med Sport. 2007;10:259-62.

16. Periard JD, Racinais S, Sawka MN. Adaptations and mechanisms of human heat acclimation: applications for competitive athletes and sports. Scand J Med Sci Sports. 2015;25(Suppl 1):20-38.

17. Ernst E, Strziga P, Schmidlechner C, Magyarosy I. Sauna effects on hemorheology and other variables. Arch Phys Med Rehabil. 1986;67(8):526-9. 
18. Garrett AT, Rehrer NJ, Patterson MJ. Induction and decay of short-term heat acclimation in moderately and highly trained athletes. Sports Med. 2011;41(9):757-71.

19. Periard JD, Travers GJS, Recinais S, Sawka MN. Cardiovascular adaptations supporting human exercise-heat acclimation. Auton Neurosci Basic Clin. 2016;196:52-62.

20. Lorenzo S, Halliwill JR, Sawka MN, et al. Heat acclimation improves exercise performance. J Appl Physiol. 2010;109(4):1140-7.

21. Pallubinsky, et al. Thermophysiological adaptations to passive mild heat acclimation. Temperature. 2017;4(2):176-86. https:// doi.org/10.1080/23328940.2017.1303562.

22. Zurawlew MJ, Mee JA, Walsh NP. Post-exercise hot water immersion elicits heat acclimation adaptations in endurance trained and recreationally active individuals. Front Physiol. 2018;9:1824. https://doi.org/10.3389/fphys.2018.01824.

23. Guy JH, Deakin GB, Edwards AM, et al. Adaptation to hot environmental conditions: an exploration of the performance basis, procedures and future directions to optimise opportunities for elite athletes. Sports Med. 2015;45(3):303-11.

24. Ammer K. The Glamorgan Protocol for recording and evaluation of thermal images of the human body. Thermol Int. 2008;18(4):125-9.

25. Moreira DG, et al. Thermographic imaging in sports and exercise medicine: a Delphi study and consensus statement on the measurement of human skin temperature. J Therm Biol. 2017;69:155-62.

26. Dill DB, Costill DL. Calculation of percentage changes in volumes of blood, plasma, and red cells in dehydration. J Appl Physiol. 1974;37:247-8.

27. Taylor NA, Machado-Moreira CA. Regional variations in transepidermal water loss, eccrine sweat gland density, sweat secretion rates and electrolyte composition in resting and exercising humans. Extrem Physiol Med. 2013;2(1):4. https://doi. org/10.1186/2046-7648-2-4.

28. Candas V, Libert JP, Vogt JJ. Human skin wettedness and evaporative efficiency of sweating. J Appl Physiol. 1979;46:522-8.

29. Kondo N, Tominaga H, Shibasaki M, Aoki K, Koga S, Nishiyasu T. Thermoregulatory adaptation in humans and its modifying factors. J Physiol. 1999;515(Pt 2):591-8.

30. Ravanelli, et al. Maximum skin wettedness after aerobic training with and without heat acclimation. Med Sci Sports Exerc. 2018;50(2):299-307. https://doi.org/10.1249/MSS.0000000000 001439.

31. Tyler CJ, Reeve T, Hodges GJ, Cheung SS. The effects of heat adaptation on physiology, perception and exercise performance in the heat: a meta-analysis. Sports Med. 2016;46:1699. https:// doi.org/10.1007/s40279-016-0538-5.

32. Rahimi GRM, Albanaqi AL, Van der Touw T. Smart NA physiological responses to heat acclimation: a systematic review and meta-analysis of randomized controlled trials. J Sports Sci Med. 2019;18(2):316-26.

33. Willmott AG, Gibson OR, Hayes M, et al. The effects of single versus twice daily short term heat acclimation on heat strain and $3000 \mathrm{~m}$ running performance in hot, humid conditions. J Therm Biol. 2016;56:59-67.

Publisher's Note Springer Nature remains neutral with regard to jurisdictional claims in published maps and institutional affiliations. 